\title{
Invarianza multicultural y validación del índice de fortaleza mental en deportistas norteamericanos y mexicanos \\ Multicultural Invariance and Validation of the Mental Toughness Index in North American and Mexican Athletes
}

*Andreas Stamatis, **Grant B. Morgan, ***Pedro Julián Flores-Moreno, ***Lenin Tlamatini Barajas -Pineda, ***Adriana Isabel Andrade Sánchez, ***Sarita Salgado Torres

*SUNY Plattsburgh (USA), ${ }^{* *}$ Department of Educational Psychology, Baylor University (USA), ${ }^{* * *}$ Universidad de Colima (México)

Resumen. La fortaleza mental (MT) es un tema ampliamente indagado y relacionado con profesiones, personalidades y la actividad deportiva. El trabajo del psicólogo del deporte es apoyar en el manejo de emociones, el afrontamiento a estresores y establecimiento de metas, haciendo uso de las características multiculturales de los deportistas. Se examinó la invariancia de MT entre atletas estadounidenses y mexicanos a través de la prueba de invarianza, mediante análisis factorial confirmatorio de grupos múltiples con modelos cada vez más restrictivos. El ajuste de los datos del modelo en ambas muestras fue satisfactorio $($ CFI mexicano $=.988$, RMSEA mexicano $=.085 ;$ CFI US $=.998$, RMSEA US $=.032)$. El modelo de invariancia escalar se seleccionó como el mejor ajuste (escalar CFI $=.981$, escalar RMSEA $=.077$ ). Los resultados implican que el significado del constructo es igual en ambas culturas y sus puntajes pueden compararse directamente.

Palabras clave: fortaleza mental, invarianza multicultural, psicología del deporte.

\begin{abstract}
Mental toughness (TM) is a widely investigated topic related to professions, personalities and sports activity. The work of the sports psychologist is to support the management of emotions, coping with factors and setting goals, making use of the multicultural characteristics of athletes. The invariance of TM was examined between American and Mexican athletes through the invariance test. It was performed by confirmatory factor analysis of multiple groups with increasingly restrictive models. The fit of the model data in both samples was very good (Mexican CFI $=.988$, Mexican RMSEA $=.085$; US CFI $=$ .998 , US RMSEA $=.032)$. The scalar invariance model was selected as the best fit $($ CFI scalar $=.981$, RMSEA scalar $=.077)$. The results imply that the meaning of the construct is the same in both cultures and its scores can be directly compared.
\end{abstract}

Keywords. mental toughness, multicultural invariance, sports psychology.

\section{Introducción}

Se ha logrado identificar que se está prestando atención al concepto de fortaleza mental (MT, por sus siglas en inglés) en varios ambientes competitivos y estresantes de la vida diaria, como los son negocios (e.g. altos directivos que tienen un MT más alto que los de nivel bajo Marchant et al., 2009), académicos (e.g. estudiantes con una alta MT tienen un mayor rendimiento, a diferencia de aquellos que registran una baja MT; Crust, Clough, Earle, Nabb, \& Clough, 2012), militares (e.g. la MT es útil en el entrenamiento antes de la batalla; Walker, Lennemann, McGregor, Mauzy \& Zupan, 2011) y médicos (e.g. la MT es descrita como un factor psicológico importante para sobrevivir al cáncer; Rom, Miller \&

Fecha recepción: 05-04-20. Fecha de aceptación: 06-08-21

Pedro Julián Flores Moreno

pedrojulian_flores@ucol.mx
Peluso, 2009). Además, es importante considerar que, en el ámbito deportivo la personalidad del deportista, su experiencia competitiva y el deporte que practica, viene a ser un determinante en la selección de las estrategias de afrontamiento motivacional y emocional, (González \& Valadez, 2016). En tanto, el deporte aporta múltiples beneficios a nivel psicológico-emocional al fomentar la autodisciplina, resolución de conflictos, regulación emocional entre otras. Es por ello que, la relación entre las habilidades cognitivas y la práctica deportiva adquirida son un objeto de análisis en la neuropsicología y psicología del deporte (Carbonell, et al., 2021). Es debido a la utilidad que han reportado diversas investigaciones, que surgió la necesidad de dirigir esta investigación sobre la MT en el deporte.

Por más de dos décadas, la MT ha sido ampliamente investigada y usada en el contexto del deporte (Gucciardi, 2017), no obstante, esta popularidad no ha sido acompañada por un consenso sobre qué es la MT y lo que no es (Anantasagar, Abbott, Stamatis \& Papadakis, 
2018; Gucciardi, 2018; Lin, Mutz, Clough \& Papageorgiou, 2017). Aunque en las últimas conceptualizaciones del término, ha dado un giro con menor variación hacia los significados (Cowden, 2017) y los planteamientos de aquellos investigadores que pudieran aun carecer de las evidencias y valor propio en cada cultura en específico (Eubank, Nesti \& Littlewood, 2017).

De acuerdo con Kagitcibasi \& Berry (1989), el trabajo psicológico en contexto multicultural puede ser agrupado en dos enfoques principales: centrarse en una sola cultura para entender lo local, lo nativo, los fenómenos ético psicológicos o para comparar entre distintas culturas entre sí y comprender de manera más amplia los patrones de relación entre las variables culturales (Allport, 1937; Pike, 1967). Para poder investigar la relación entre cultura y comportamiento, fue necesario llevar a cabo este estudio de MT en el deporte, el cual se basó en enfoques nomotéticos y aproximaciones éticas.

Es debido a la disparidad y confusión en la operatividad de la MT la importancia de crear y mantener temas tanto en investigación (Weinberg, Freysinger \& Mellano, 2018), como en la práctica (Stamatis, Robinson \& Morgan, 2018) y no solo en la abundancia de definiciones ${ }^{1}$ (Clough, Earle \& Sewell, 2002; Gucciardi, Gordon \& Dimmock, 2008; Jones, Hanton \& Connaughton, 2002), pero sí en inventarios y/o cuestionarios, lo cual es una indicación de las aún existentes discrepancias de asuntos cruciales en la comunidad científica, tales como, si un modelo Uni - o Multidimensional debiera ser adoptado para conceptualizar la MT basándose en las propiedades psicométricas de varios de esos instrumentos, en donde los resultados del análisis del estudio de la confiabilidad de la MT y de la viabilidad de sus conclusiones aún se encuentran en proceso (Gucciardi, 2017, 2018).

El instrumento de medición denominado, Índice de Fortaleza Mental (por sus siglas MTI), fue diseñado al conceptualizar el término como unidimensional y estructurado por «una caravana de recursos personales» (Gucciardi, Hanton, Gordon, Mallett \& Temby, 2015, p.30), la cual consiste en grupo de ocho elementos, don-

Tabla 1

\begin{tabular}{ll} 
Los recursos personales evaluados por item en MTI & \\
\hline Item & Recurso personal \\
\hline 1. Creo en mi capacidad de lograr mis metas & Auto eficiencia generalizada \\
2. Soy capaz de regular mi concentración cuando realizo alguna tarea & Regulación de las emociones \\
3. Soy capaz de usar mis emociones para hacer las cosas como lo deseo & Regulación de la atención \\
4. Lucho por el éxito continuo & Mentalidad de éxito \\
5. Ejecuto mi conocimiento de lo que sea requerido para lograr mis metas & Fortaleza \\
6. Consistentemente venzo la adversidad & Vencer la adversidad \\
7. Soy capaz de desempenar habilidades adecuadas o conocimientos cuando & Conocimiento en contexto \\
soy retado & Estilo optimista \\
8. Busco lo positivo en la mayoría de las situaciones &
\end{tabular}

de cada ítem que describe la MTI corresponde a un recurso de MT en específico (Tabla 1). Como referencia a resaltar, el MTI fue desarrollado y validado en Australia, utilizando participantes provenientes y no provenientes del contexto deportivo.

Recientemente, en un esfuerzo por revelar la importancia cultural mundial de la MT, Gucciardi, Zhang, Ponnusamy, Si \& Stenling (2016) reportaron una invarianza intracultural del MT al emplearlo en deportistas, donde los hallazgos indicaron el mismo factor latente y unidimensional, así como un significado que es atribuido a los grupos construidos en Australia, Malasia y China. En otras palabras, esta configuración unidimensional es una descripción sostenible de la MT para esas culturas. De este modo, la versión inglesa, malasio y china del MTI puede ser utilizada en futuras investigaciones relacionadas con la MT. Es desde entonces que, las propiedades psicométricas y utilidad del MTI han sido explorada nuevamente en países como China utilizando estudiantes y deportistas, en ambos casos, de nivel universitario (Li, Zhang \& Zhang, 2017), así como también en África del Sur con jugadores de tenis (Cowden, 2018).

Con respecto al proyecto chino, los hallazgos respaldan tanto la conceptualización unidimensional del conocimiento, así como su uso. Es así, como se encontró que el MTI es internamente confiable cuando se evalúan a atletas sudafricanos de un deporte en específico (Cowden, 2018). Por consiguiente, los resultados previeron un mayor apoyo al utilizar el MTI como una medida unidimensional de la MT. Aun así y, de cualquier modo, es necesario realizar el análisis psicométrico para que el MTI sea utilizado especialmente en culturas deportivas donde la MT es muy popular (e.g. Estados Unidos y países europeos), situación que aún no es comúnmente realizada.

Por lo tanto y debido a lo anterior, el propósito de esta investigación fue dual: (a) investigar la invarianza multicultural de la fortaleza mental entre dos grupos culturales de atletas, (norteamericanos y mexicanos) y (b) validar el MTI al idioma español.

\section{Método}

Se realizó un estudio de tipo no experimental con un enfoque descriptivo, debido a que el objeto de estudio se basó en la definición y/o la categorización de la MT en un grupo de deportistas con diferente dialecto y con ello llevar acabo la validación del MTI al idioma español (Ato, López \& Benavente, 2013). El tipo de 
muestreo aplicado fue no probabilístico por invitación e inclusión de voluntarios, basando su procedimiento en la búsqueda de casos que se encontraran en un ambiente o contexto deportivo (Hernández, Fernández \& Baptista, 2010). Como criterios de elegibilidad se consideró intervenir en sujetos que estuvieran activos y pertenecieran a un equipo deportivo, además que presentaran una mayoría de edad. Derivado de ello, se logró la participación de 171 atletas mexicanos y 173 atletas norteamericanos. De aquellos que reportaron el sexo, aproximadamente en la muestra norteamericana la mitad fueron masculinos y la otra mitad femeninos, mientras que en la muestra mexicana predominaron los masculinos. Además, algunos deportes fueron representados sólo por norteamericanos (e.g. béisbol, golf, y softbol) y otros solo por mexicanos (e.g. ciclismo, crossfit, gimnasia, balonmano, artes marciales, levantamiento de pesas, rugby y luchas) (Tabla 2).

\section{Procedimiento}

El levantamiento y análisis de datos tuvo una duración tres meses, donde se emplearon dos fases: en la primera se llevó a cabo la traducción del instrumento y validación intercultural, la cual fue generada y basada en las recomendaciones de Hambleton \& Kanjee (1995) utilizándose una transcripción de ida y vuelta a partir del MTI original, donde, interpretes bilingües independientes con la certificación C1 Advanced del sistema Cambrige Assesment English ${ }^{\circledR}$ vigente estuvieron involucrados, con ello se logró obtener como producto la versión al español con población mexicana del MTI. En la segunda fase, los investigadores contactaron a entrenadores y deportistas, dándoles los detalles acerca del propósito del estudio, los beneficios y riesgos asociados. A su vez, los entrenadores comunicaron los propósitos de la investigación a sus jugadores y/o atletas.

Para el caso de la muestra norteamericana, se envió un link a través del correo electrónico provisto por los atletas a los investigadores, con el cual fue posible contestar el instrumento de manera digital. Para mantener los procedimientos éticos de esta investigación, se obtuvo de forma electrónica un permiso de consentimiento informado de todos los participantes incluidos en el estudio. Para la muestra mexicana se siguió el mismo procedimiento de acercamiento con entrenadores y deportistas. Mientras que, para la aplicación del instrumento previamente traducido al español, se visitaron las diferentes instalaciones deportivas donde se encontraban los equipos deportivos, de esta manera fue administrado a los deportistas a través de copias impre- sas en papel, que además incluía una declaración de consentimiento informado.

\section{Descripción del instrumento de medición}

El MTI está conformado por ocho ítems con una escala Likert, con respuestas de siete puntos $(1=$ Falso, el $100 \%$ de las veces y $7=$ verdadero, el $100 \%$ de las veces). Los puntajes del MTI han sido reportados como recurrentemente confiables y sus inferencias válidas (Cowden, 2018; Gucciardi et al., 2015; Gucciardi et al., 2016; Li et al., 2017). Derivado de su confiabilidad (Anexo 1).

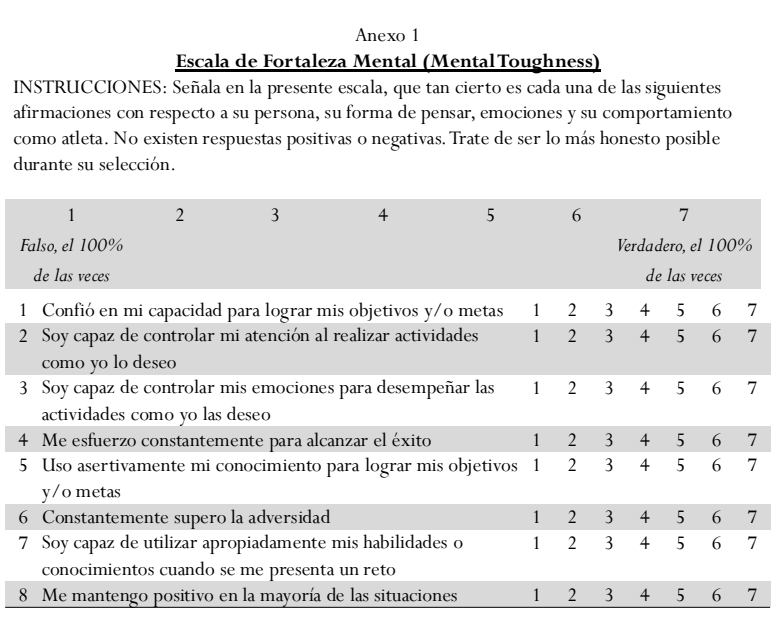

\section{Análisis estadístico}

Se aplicó estadística descriptiva para cada ítem de la muestra. Se empleó la prueba de invarianza, la cual fue llevada a cabo utilizando análisis factorial confirmatorio de múltiples grupos con modelos cada vez más restrictivos, donde, primeramente, se adaptó un modelo unidimensional a cada muestra para asegurar que fuera un modelo adecuado de información ajustada. Como segundo paso, se estimaron y configuraron (número igual de dimensiones), las métricas (configurabilidad + carga igual) y los modelos de invarianza escalar (métrica cortes iguales). El proceso para adaptar los modelos fue cada vez más restrictivo y se llevó como parte de la examinación de las medidas de la propia invarianza (Brown, 2015; Putnick \& Bornstein, 2016; Vandenberg \& Lance, 2000; Widaman \& Reise, 1997). La invarianza escalar fue del tipo mínimo de invarianza, la cual infirió igualdad multicultural.

Para evaluar la adecuación del modelo de datos, se utilizó el índice de ajuste comparativo (CFI por sus siglas en inglés), error cuadrático medio de la aproximación (RMSEA por sus siglas en inglés), error cuadrático medio residual estandarizado (SRMR por sus siglas en inglés), donde las diferencias entre estos índices en los 
modelos fueron, para los valores del CFI en o sobre el .95 y RMSEA en o por debajo de .05. Por su parte, el CFI se estimó entre .90 y .95 , mientras que el RMSEA se calculó entre .05 y .08. Los valores de SRMR se calcularon en o por debajo de .08. El ajuste gradual también fue examinado al grabar los cambios en CFI, cambiado $\mathrm{d} \gg .02$, los cuales no se consideraron perjudiciales en el modelo de ajuste incremental (Cheung \& Rensvold, 2002). Todos los análisis fueron llevados a cabo en $R$ (versión 3.4.0, R CoreTeam, 2017) empleando los paquetes de psych y lavaan (Rosseel, 2012).

Con respecto al enfoque estadístico, Gucciardi et al., (2016) usó la estimación Bayesian, sin embargo, optó por estimar que todos los modelos utilizando tuvieran el estimador de mínimos cuadrados ponderados con media y ajuste en varianza (WLSMV por sus siglas en inglés). Este estimador ha mostrado rendir paramétricamente y estandarizar el error estimando con un sesgo moderado en todas las muestras pequeñas y modelos grandes, aun en presencia de la información anormal y la ordenada categóricamente (Bandalos, 2008, 2014; DiStefano, 2002; DiStefano \& Morgan, 2014; Flora \& Curran, 2004; Li, 2016a, 2016b; Rhemtulla, Brosseau-Liard \& Savalei, 2012).

\section{Resultados}

En la muestra norteamericana, el atletismo fue el deporte con más representatividad $(n=39,22.6 \%)$ seguida de fútbol americano $(n=32,18.5 \%)$, béisbol $(n=21,12.1 \%)$, y fútbol $(n=19,11.0 \%)$. Mientras que en la muestra mexicana fue, el fútbol el deporte más representado $(n=60,35.1 \%)$, seguido del balonmano $(n=23,13.5 \%)$, y el baloncesto $(n=16$, 9.4\%). La edad promedio en la muestra norteamericana es ligeramente más baja $\left(M_{\text {edad }}=20.0, S D=1.4\right)$ que en la muestra mexicana $\left(M_{\text {edad }}=20.2, S D=2.1\right)$. El centro de este estudio fue el MT en deportes en general, así que, los deportes específicos representados a través de las muestras no fue una variable de control incluida. Por lo tanto, la popularidad de los deportes específicos varío entre culturas y los deportes representados fueron un reflejo de las diferencias entre las mismas. Tabla 2.

Los resultados descriptivos para cada ítem y dentro de cada muestra aparecen en la tabla 3, donde, generalmente las respuestas por ítem indicaron niveles altos de MT a través de ambas muestras. En el cual, la muestra norteamericana, el ítem con la media más alta fue «Yo lucho por éxito continuo,» mientras que, en la
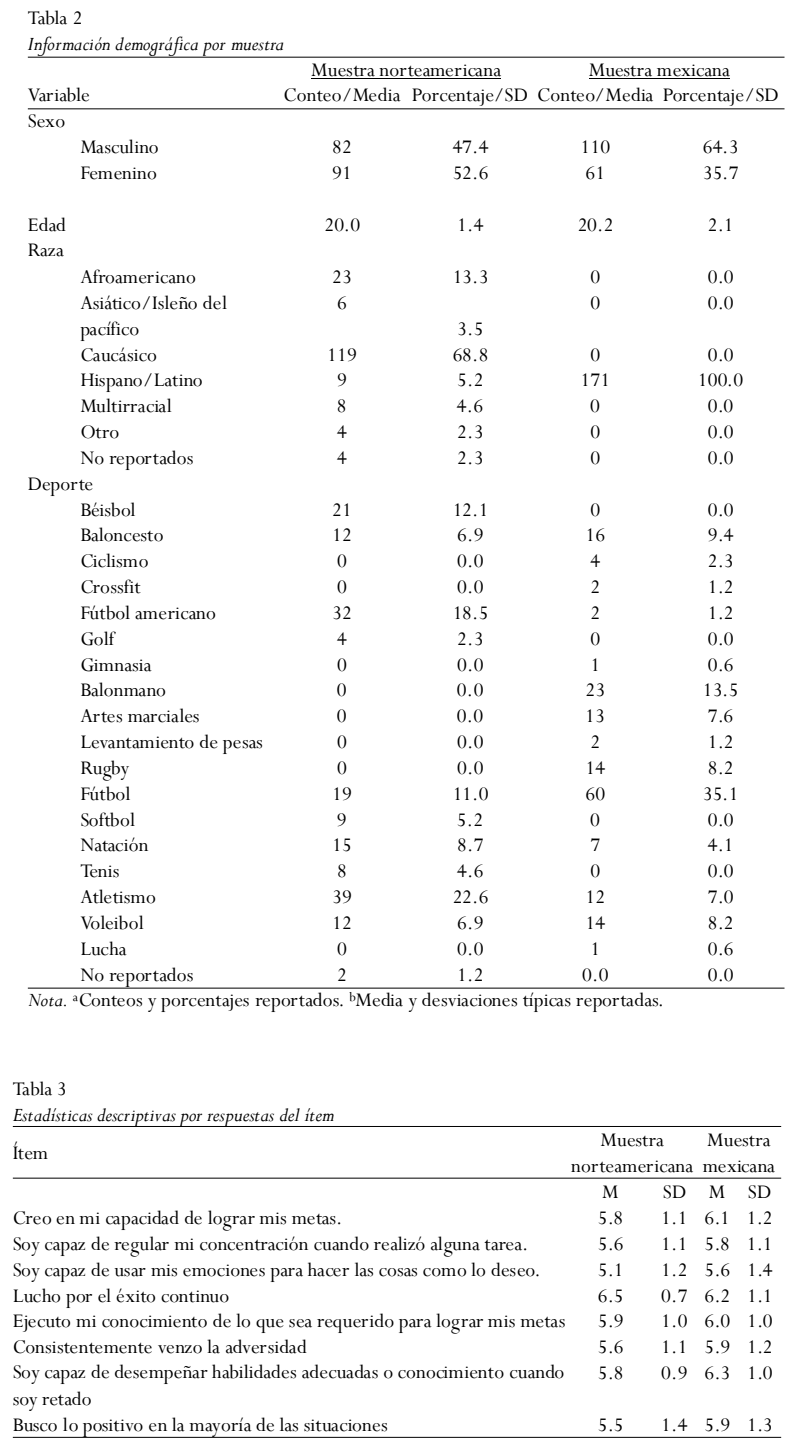

muestra mexicana, fue «Soy capaz de desempeñar habilidades adecuadas o conocimiento cuando soy retado».

En lo que refiere al factor de la estructura, un modelo unidimensional fue primeramente considerado para cada muestra, el ajuste del modelo fue adecuado en ambas muestras; el ajuste indicó que en la muestra estadounidense el CFI fue $=.998$, el RMSEA $=.032(.000, .081)$, y el SRMR $=.053$; mientras que en la muestra mexicana el CFI fue $=.988$, el RMSEA $=.085(.051, .119)$, y el SRMR $=.064$. Luego, el modelo fue estimado simultáneamente en ambas muestras (e.g. invarianza configurativa) y de nuevo el modelo logró una adecuada información ajustada $(\mathrm{CFI}=.993, \mathrm{RMSEA}=.067[.040$, .092] y SRMR $=.056$ ).

El modelo de invarianza métrica logró un patrón de información ajustada aceptable y el CFI estimó el cambio muy por debajo del corte preestablecido de .02. El ajuste en el modelo de invarianza métrica fue $\mathrm{CFI}=$ .987, RMSEA $=.087$, SRMR, .071, CFI $=.006 . \mathrm{El}$ 
ajuste en el modelo de invarianza escalar fue también aceptable $(\mathrm{CFI}=.91$, RMSEA $=.077$, SRMR, .061, $\mathrm{CFI}=.006)$. Dado que la invarianza escalar fue respaldada por las dos muestras, el producto subyacente de la MT fue posible. Por lo tanto, el promedio de la MT en la muestra mexicana fue restringida a cero para poder permitir las comparaciones con la muestra de los Estados Unidos. El valor de la MT en la muestra estadounidense, en promedio fue de .45 más baja que la muestra mexicana, lo cual indica que los atletas mexicanos reportaron una MT más alta por un medio en la desviación estándar. El modelo final seleccionado se muestra en la figura 1.

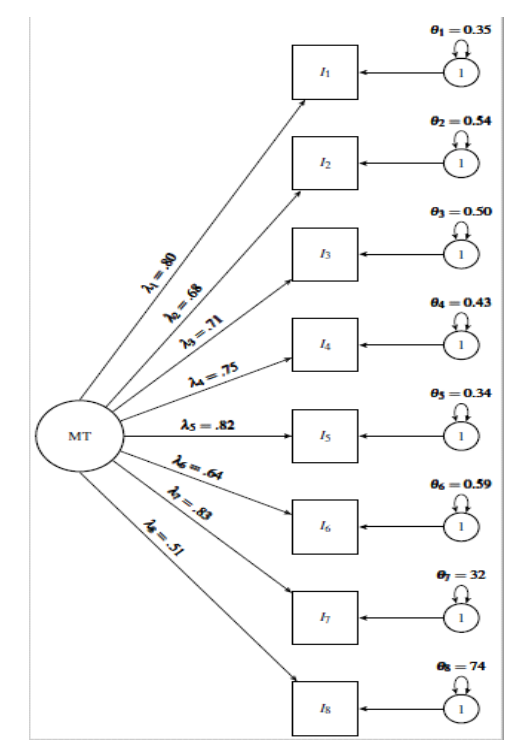

Figura 1. Trayectoria del modelo de MTI para población de deportistas mexicanos

Figura 1. El modelo final elegido fue medir la invarianza entre las muestras estadounidenses y mexicana (se muestran estimaciones sin estandarizar). En la figura ?k representa el valor de carga entre el ítem $k$ y MT, y ? $k$ representa la varianza residual del ítem $k$. Las estimaciones de parámetros mostrados en negritas, son aquellas que son invariantes entre las muestras (i.e., el valor fue el mismo en las muestras mexicana y estadounidenses) el parámetro estimado con $M$ or US subíndice aplicando solo la para las muestras mexicanas y estadounidenses, respectivamente. Por ejemplo, ? $1=0.80$ indica que la carga por ítem 1era .80 para ambas muestras mexicanas y estadounidenses. Los límites de categoría por item no fueron incluidos para darle claridad a la figura; los límites fueron invariantes entre las muestras.

\section{Discusión}

La MT es extensivamente usada en ámbitos deportivos en países de Europa y Estados Unidos. Desde su desarrollo en el 2015, el MTI ha sido comúnmente elegido como un instrumento de medición para este constructo en las dos regiones geográficas previamente mencionadas. Por tanto, derivado de la necesidad de conocer el uso y aplicabilidad, se han realizado esfuerzos para ofrecer avances que muestran la función principal y el valor cultural que funge la MT durante la aplicación del MTI en países como Australia, China, Malasia y África del Sur (Gucciardi, et al., 2016). Además, la evaluación de la literatura, sugiere que la MT puede llegar a representar un indicador positivo de la salud mental, al estar asociada con el logro de una meta, mejora del desempeño, la obtención de objetivos, entre otros aspectos en diferentes tipos de población, incluidos los deportistas (Gucciardi, Hanton \& Fleming, 2016). Es por ello que, extraído del enfoque nomotético (Allport, 1937) y de los aspectos generales culturales del comportamiento (Pike, 1967), el propósito de este estudio fue examinar la invarianza multicultural de la Fortaleza Mental entre un grupo de deportistas Norteamericanos y Mexicanos así como también la validación del MTI al idioma español.

Desde el punto de vista teórico, los resultados (a) más allá de la puesta en marcha de la MT al respaldar su unidimensionalidad y (b) la validez psicométrica del MTI al agregar al constructo existente evidencia validable (Cowden, 2018; Gucciardi et al., 2015; Gucciardi et al., 2016; Li et al., 2017), desde una perspectiva práctica, donde los hallazgos indican que la versión mexicana del MTI puede ser usada para medir la MT en México y otros países de América Latina, como lo expuesto por Gucciardi et al., (2016) quienes evaluaron la invariancia intercultural en tres países diferentes, identificando que, para los grupos estudiados existe una invariancia métrica, escalar aproximada y que los hallazgos reportados muestran la necesidad de realizar los ajustes culturales necesarios a la medición de la MT para captar con una mayor amplitud de los aspectos contextuales. Mientras que Li, et al., (2017) compararon deportistas y estudiantes de nivel universitario, en ambos casos. Resaltando que la MT se asoció positivamente con las necesidades psicológicas básicas y el esfuerzo realizado en deportistas mientras que en los estudiantes se relacionó en mayor medida con la satisfacción por la vida.

Es posible aceptar que la MT es un indicador asociado al logro de objetivos y metas, que ésta puede llegar a variar, pues la diversidad de deportes presentados en ambas muestras puede ser considerado como una fortaleza de este estudio, ya que se ha identificado que el nivel de MT se asocia a las características demográficas y deportivas, permitiendo que el entrenamiento se pueda dirigir al desarrollo de habilidades mentales para la mejora del desempeño deportivo (Zeiger \& Zeiger, 2018). No obstante, a pesar de que las muestras no fueron homogéneas, los resultados no mostraron una invarianza cultural que indicara que los hallazgos pudieran ser generalizados en más deportes, por tanto, la versión del MTI en idioma español puede ser aplicada en todas las actividades deportivas y atletas como un instrumento de diagnóstico y control del desempeño 
deportivo.

Por falta de investigación relevante, los resultados no pueden ser comparados en gran medida con estudios de medidas equivalentes a otras herramientas de medición de MT. No obstante, su utilidad se explica en estudios como los realizados por Gómez-López, GranerGallegos \& Folgar, (2013), quienes analizaron los factores psicológicos que afectan a los piragüistas de alto rendimiento con el Inventario Psicológico de Ejecución Deportiva (IPED), el cual resalto que los sujetos evaluados poseen un alto nivel motivacional, además de confirmarse que el instrumento utilizado demuestra una validez y fiabilidad que es aceptable para la evaluación en este tipo de deportistas. Así mismo, el uso de instrumentos como el IPED, logran describir la habilidad del deportista para mantenerse de forma consiente en el estado ideal de desempeño físico mientras se encuentra en competencia, y con ello disminuir factores que limiten su óptimo rendimiento (Campos-Salinas, Berengüí \& Hernández-Mendo, 2019). En este sentido otros inventarios o cuestionarios han sido validados con éxito en relación a la efectividad colectiva en el contexto del deporte en una muestra mexicana, (Martínez-Alvarado, Guillen, Aguiar \& Magallanes, 2020), mas no abordan el contexto de la MT, sus características y dimensiones. Sin embargo, en general, el propósito del estudio fue logrado, por lo que es necesario resaltar que, 173 norteamericanos que completaron la versión original en inglés del MTI y 171 mexicanos que completaron el equivalente en español, presentaron información de un modelo reportado como satisfactorio (CFI mexicana $=$ .995 , RMSEA mexicana $=.095$; CFI Norteamericana $=.998$, RMSEA Norteamericana $=.032$ ) además un modelo de invarianza escalar que fue seleccionado como el mejor ajustado (CFI escalar $=.981$, RMSEA escalar $=.077$ ).

\section{Conclusión}

El resultado de este análisis provee evidencia para la invarianza escalar parcial del MTI utilizado en muestras de deportistas norteamericanas y mexicanas. Esto implica que el significado del constructo y los niveles de items subyacentes son iguales en ambas culturas. Por tanto, ambas pueden ser directamente comparadas en sus puntajes en la variable latente, por ejemplo, si un atleta norteamericano y un mexicano tiene un puntaje promedio de 5.5, indicaría que los dos atletas tienen casi la misma cantidad de MT.

\section{Limitaciones}

Este estudio no está exento de limitaciones, primero, aunque los autores siguieron el mismo procedimiento, la recolección de información fue de manera electrónica en los Estados Unidos y en papel por medio de fotocopias en México, quizás pudo haber afectado la cantidad de (a) información registrada incorrectamente o (b) error en la captura de datos, no obstante, estos aspectos se cuidaron en todo momento con el propósito de evitar sesgos y errores. Segundo, aunque ambas muestras fueron independientes (e.g. las muestras fueron diferentes y no tuvieron influencia una en la otra), hubo algunas diferencias en las variables demográficas, por mencionar algunas, en la muestra mexicana, se presentó una mayor proporción de participantes masculinos, además, todos los participantes se identificaron como hispanos/latinos, sin embargo y de cualquier modo a pesar de la diferencia demográfica, la robusta evidencia presentada de invarianza entre ambos grupos fue reportada favorable. Tercero, los deportes representados entre las dos muestras fueron desiguales (tabla 1) y a pesar de estas diferencias, la evidencia de invarianza entre las muestras concurrió fuerte. Cuarta, con respecto a la generalidad, los resultados son limitados en relación a los contextos no deportivos y otros países y culturas por lo que es necesario llevar a cabo la intervención del MTI en otras naciones o países.

\section{Investigaciones futuras}

Como parte final, este estudio agregó evidencia existente con respecto a la universalidad de MT en el deporte. Los autores usaron el MTI y se concentraron en investigar invarianza cultural al comparar puntajes de la MT en deportistas mexicanos y norteamericanos utilizando el MTI. Se hace el énfasis en aumentar los esfuerzos que deberán continuar con el respaldo de un enfoque culturalmente informado para comprender de mejor manera la MT al evaluar el MTI en culturas «inexploradas» (e.g. América del Sur y Central, u otros idiomas) y al investigar hasta qué punto el instrumento y la MT generalicen (e.g. ético) en deportistas o no deportistas. Por lo que las medidas equivalentes en otros dialectos del español abrirán nuevas y emocionantes puertas de investigación de la MT incrementando el entendimiento, la relevancia específica y cultural de este constructo. Como resultado, los investigadores pudieran ser capaces de avanzar hacia una claridad conceptual y consensuada, así como al desarrollo de instrumentos 
que evalúen el constructo hasta lo más profundo plenamente.

\section{Referencias}

Allport, G. W. (1937). Personality: A psychological interpretation. NewYork, NY: Henry Holt.

Anantasagar,T.,Abbott, K., Stamatis,A., \& Papadakis,Z. (2018). MentalToughness in Sport: Moving towards conceptual clarity and consensus. International Journal of Exercise Science, 2(10).

Ato, M., López - García, J. J., \& Benavente, A. (2013). Un sistema de clasificación de los diseños de investigación en psicología. Anales de Psicología / Annals of Psychology, 29(3), 1038-1059.

Bandalos, D. L. (2008). Is parceling really necessary? A comparison of results from item parceling and categorical variable methodology. Structural equation modeling, 15(2), 211-240

Bandalos, D. L. (2014). Relative performance of categorical diagonally weighted least squares and robust maximum likelihood estimation. Structural Equation Modeling: a multidisciplinary journal, 21(1), 102-116.

Brown,T.A. (2015). Confirmatory Factor Analysis for Applied Research. New York, NY:The Guilford Press.

Campos-Salinas, J.A., Berengüí, R., \& Hernández-Mendo,A. (2019). Inventario Psicológico de Ejecución Deportiva para Árbitros (IPEDA): Adaptación y Propiedades de Medida. Revista Iberoamericana de Diagnóstico y Evaluacióne Avaliação Psicológica, 4(53), 141-152.

Carbonell, N., Hernández-Prados, M. Á., Sarmiento, B. R., González, E. M.,Aguaded, M.C. \& Álvarez, J.A.(2021). Funciones ejecutivas y rendimiento futbolístico. Diseño y evaluación de un programa de intervención. Retos:nuevas tendencias en educación física, deporte y recreación, 42, 306315.

Cheung, G. W., \& Rensvold, R. B. (2002). Evaluating goodness-of-fit indexes for testing measurement invariance. Structural equation modeling, 9(2), 233-255.

Clough, P., Earle, K., y Sewell, D. (2002). Mental toughness: The concept and its measurement. In I. M. Cockerill (Ed.), Solutions in sport psychology (pp. 32-43). Longon, England: Thompson.

Cowden, R. G. (2017). Mental toughness and success in sport: A review and prospect. Open Sports Sciences Journal, 10. doi:10.2174/1875399X01710010001.

Cowden, R. G. (2018). MentalToughness Inventory: Factorial validity and ethnic group measurement equivalence in competitive tennis. Current Psychology, 1-6. doi:10.1007/ s12144-018-9798-6.

Crust,L., Clough, P., Earle, K., Nabb, S., \& Clough,A. (2012). From the sports field to the classroom: relationships between mental toughness, achievement, and progression in first-year university sports students. Paper presented at the Back to the Future: Reflections and Implications Following the 2012 Olympic and Paralympic Games: BPS Nor th East and Nor thWest of England Conference Manchester, England.

DiStefano, C. (2002). The impact of categorization with confirmatory factor analysis. Structural equation modeling, 9(3), 327-346.

DiStefano, C., \& Morgan, G. B. (2014). A comparison of diagonal weighted least squares robust estimation techniques for ordinal data. Structural Equation Modeling: a multidisciplinary journal, 21(3), 425-438.

Eubank, M., Nesti, M., \& Littlewood, M. (2017).A culturally informed approach to mental toughness development in high performance sport. International Journal of Sport Psychology, 48(3), 206-222.

Flora, D. B., \& Curran, P. J. (2004). An empirical evaluation of alternative methods of estimation for confirmatory factor analysis with ordinal data. Psychological methods, 9(4), 466.

Gómez-López, M., Granero - Gallegos, A., \& Folgar, M. I. (2013).Análisis de los factores psicológicos que afectan a los piragüistas en el alto rendimiento. Revista Iberoamericana de Diagnóstico y Evaluación-e Avaliação Psicológica, 1(35), 57-76.

González, J. \& Valadez,A. (2016). Personalidad y respuesta psicológica en deportistas. Representación temporal y adaptativa del proceso persona-deporte. Retos:nuevas tendencias en educación fisica, deporte y recreación, (30), 211 215.

González, J. \& Valadez,A. (2016). Personalidad y respuesta psicológica en deportistas. Representación temporal y adaptativa del proceso persona-deporte. Retos:nuevas tendencias en educación física, deporte y recreación, (30), 211 215.

Gucciardi, D. F. (2017). Mental toughness: progress and prospects. Current Opinion in Psychology, 16, 17-23. doi:10.1016/j.copsyc.2017.03.010.

Gucciardi, D. F. (2018). Commentary: Mental toughness and individual differences in learning, educational and work performance, psychological well-being, and personality: A systematic review. Frontiers in psychology, 8, 2329.

Gucciardi,D. F., Gordon, S., \& MentalToughness in Australian Football. Journal of Applied Sport Psychology, 20(3), 261281.

Gucciardi, D. F., Hanton, S., \& Fleming, S. (2016).Are mental toughness and mental health contradictory concepts in elite sport? A narrative review of theory and evidence. Journal of Science and Medicine in Sport, 20(3), 307-311.

Gucciardi, D. F., Hanton, S., Gordon, S., Mallett, C.J., \& Temby, P. (2015). The Concept of Mental Toughness: Tests of Dimensionality, Nomological Network, and Traitness. 
Journal of Personality, 83(1), 26-44. doi:10.1111/ jopy. 12079.

Gucciardi,D.F., Zhang, C.-Q., Ponnusamy,V., Si, G., \& Stenling, A. (2016). Cross-cultural invariance of the mental toughness inventory among Australian, Chinese, and Malaysian athletes: a bayesian estimation approach. Journal of Sport and Exercise Psychology, 38(2), 187-202.

Hambleton, R. K., \& Kanjee,A. (1995). Increasing the validity of cross-cultural assessments: Use of improved methods for test adaptations. European Journal of Psychological Assessment, 11(3), 147-157.

Hernández, R., Fernandez, C., \& Baptista, P., (2010). Metodología de la investigación. Mc Graw Hill. México.

Jones, G., Hanton, S., \& Connaughton, D. (2002). What is this thing called mental toughness? An investigation of elite sport performers. Journal of Applied Sport Psychology, 14(3), 205-218. doi:10.1080/10413200290103509.

Kagitcibasi, C., \& Berry, J. W. (1989). Cross-cultural psychology: Current research and trends. Annual review of psychology, 40(1), 493-531.

Li, C. - H. (2016a). Confirmatory factor analysis with ordinal data: Comparing robust maximum likelihood and diagonally weighted least squares. Behavior Research Methods, 48(3), 936-949.

Li, C., Zhang, C. Q., \& Zhang, L. (2017). Further Examination of the Psychometric Properties of the Mental Toughness Inventory: Evidence from Chinese Athletes and University Students. Current Psychology, 1-7. doi:10.1007/s12144017-9692-7.

Li, C.H. (2016b). The performance of ML, DWLS, and ULS estimation with robust corrections in structural equation models with ordinal variables. Psychological methods, 21(3), 369.

Lin,Y., Mutz, J., Clough, P. J., \& Papageorgiou, K.A. (2017). Mental toughness and individual differences in learning, educational and work performance, psychological wellbeing, and personality: A systematic review. Frontiers in psychology, 8(AUG). doi:10.3389/fpsyg.2017.01345.

Marchant, D. C., Polman, R. C., Clough, P., Jackson, J. G., Levy, A. R., \& Nicholls, A. R. (2009). Mental toughness: Managerial and age differences. Journal of Managerial Psychology, 24(5), 428-437.

Martinez-Alvarado, J.R., Guillen, F., Aguiar, L. H., \& Magallanes, A. G. (2020). Translation andValidation of the Collective Efficacy Questionnaire for Sports (CEQS) in Mexican Athletes. Revista Iberoamericana De Diagnóstico y Evaluacion-E Avaliacao Psicologica. 1(54), 47-55.

Pike, K. L. (1967). Language in relation to a unified theory of the structure of human behaviour.The Hague, Netherlands: Mouton and Co.

Putnick, D. L., \& Bornstein, M. H. (2016). Measurement invariance conventions and reporting: The state of the art and future directions for psychological research. Developmental Review, 41, 71-90.

R Core Team. (2017). R: A language and environment for statistical computing. Vienna, Austria: R Foundation for Statistical Computing.

Rhemtulla, M., Brosseau-Liard, P.É \& Savalei,V. (2012).When can categorical variables be treated as continuous? A comparison of robust continuous and categorical SEM estimation methods under suboptimal conditions. Psychological methods, 17(3), 354.

Rom, S. A., Miller, L., y Peluso, J. (2009). Playing the game: Psychological factors in surviving cancer. International Journal of Emergency Mental Health, 11(1), 25-36.

Rosseel,Y.(2012). Lavaan:An R package for structural equation modeling and more. Version 0.5-12 (BETA). Journal of Statistical Software, 48(2), 1-36.

Stamatis,A., Robinson, E. L., \& Morgan, G. B. (2018). Mental Toughness in Collegiate Strength and Conditioning: Widely Used, Widely Misunderstood. International Research in Higher Education, 3(2), 35-50. doi:10.5430/ irhe.v3n2p35.

Vandenberg, R. J., \& Lance, C. E. (2000).A review and synthesis of the measurement invariance literature: Suggestions, practices, and recommendations for organizational research. Organizational research methods, 3(1), 4-70.

Walker,T. B., Lennemann, L. M., McGregor, J. N., Mauzy, C., \& Zupan, M. F. (2011). Physiological and psychological characteristics of successful combat controller trainees. Journal of Special Operations Medicine, 11(1), 39-47.

Weinberg, R., Freysinger, V., \& Mellano, K. (2018). How can coaches build mental toughness? Views from sport psychologists. Journal of Sport Psychology in Action, 9(1), 110.

Widaman, K. F., \& Reise, S. P. (1997). Exploring the measurement invariance of psychological instruments: Applications in the substance use domain. In K. J. Bryant, M.Windle, \& S. G.West (Eds.), the science of prevention: Methodological advances from alcohol and substance abuse research (p. 281-324). American Psychological Association.

Zeiger, J. S., \& Zeiger, R. S. (2018). Mental toughness latent profiles in endurance athletes. PLoS One, 13(2), e0193071.

\section{(Footnotes)}

${ }^{1}$ For a chronology of MT definitions, see Gucciardi (2017). 